This information is current as of April 26, 2023.

\title{
Differentiation of Brain Abscesses from Necrotic Glioblastomas and Cystic Metastatic Brain Tumors with Diffusion Tensor Imaging
}

C.H. Toh, K.-C. Wei, S.-H. Ng, Y.-L. Wan, C.-P. Lin and M. Castillo

AJNR Am J Neuroradiol 2011, 32 (9) 1646-1651

doi: https://doi.org/10.3174/ajnr.A2581

http://www.ajnr.org/content/32/9/1646 


\section{ORIGINAL RESEARCH}

C.H. Toh

K.-C. Wei

S.-H. Ng

Y.-L.Wan

C.-P. Lin

M. Castillo

\title{
Differentiation of Brain Abscesses from Necrotic Glioblastomas and Cystic Metastatic Brain Tumors with Diffusion Tensor Imaging
}

BACKGROUND AND PURPOSE: The differentiation of abscesses from glioblastomas and metastases may not always be possible on the basis of DWI. Our hypothesis was that differences in diffusion properties as detected by DTI allow differentiation of abscess from glioblastomas and metastasis. Furthermore, diagnostic performance of tensor metrics quantifying anisotropy or tensor shapes is better than that of ADC in measuring mean diffusivity for this purpose.

MATERIALS AND METHODS: DTI was performed in 15 abscesses, 15 necrotic glioblastomas, and 26 cystic metastases. In each lesion, manually segmented into 4 regions of interest (ie, cystic cavity, enhancing rim, and immediate [edema most adjacent to the enhancing rim] and distant zones of edema), $F A, A D C, C_{l}, C_{p}$ and $C_{s}$ values were measured and statistically compared among groups and evaluated with $\mathrm{ROC}$ curve analysis. The presence of a hyperintense FA rim (a rim of edematous tissue that was hyperintense on the FA map) was assessed visually.

RESULTS: Abscess was significantly different from glioblastoma for all tensor metrics measured in the cystic cavity and immediate zone of edema and for all except $C_{l}$ in the enhancing rim. Abscess was significantly different from metastasis for all tensor metrics measured in the cystic cavity and

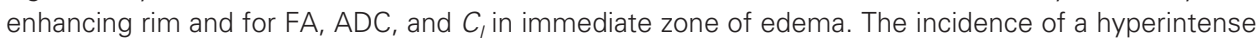
FA rim was significantly higher in glioblastoma and metastasis compared with abscess. The 3 tensor metrics with the highest performance in differentiating abscess from glioblastoma and metastasis were FA, $C_{l}$ and $C_{s}$ of the cystic cavity.

concLUSIONS: DTI is able to differentiate abscess from glioblastoma and metastasis. FA, $C_{l}$, and $C_{s}$ outperformed ADC in diagnostic performance comparisons.

\begin{abstract}
ABBREVIATIONS: $A D C=$ apparent diffusion coefficient; $A \cup C=$ area under the curve; $C C=$ cystic cavity; $C_{l}=$ linear tensor; $C_{p}=$ planar tensor; $C_{s}=$ spheric tensor; $\mathrm{DTI}=$ diffusion tensor imaging; $\mathrm{DWI}=$ diffusion-weighted imaging; $\mathrm{DZE}=$ distant zone of edema; $\mathrm{ER}=$ enhancing rim; $\mathrm{FA}=$ fractional anisotropy; GB = glioblastoma; IZE = immediate zone of edema; Mets = metastasis; MPRAGE = magnetization-prepared rapid acquisition of gradient echo; NAWM = normal-appearing white matter; $\mathrm{ROC}=$ receiver operating characteristic
\end{abstract}

$\mathrm{t}$ is difficult to differentiate necrotic glioblastomas, cystic metastases, and abscesses with conventional MR imaging. All can appear as expansile rim-enhancing masses with prominent perifocal edema. DTI is a noninvasive imaging technique

Received January 19, 2011; accepted after revision February 6.

From the Departments of Medical Imaging and Intervention (C.H.T, S.-H.N, Y.-L.W) and Neurosurgery (K.-C.W), Chang Gung Memorial Hospital and Chang Gung University College of Medicine, Tao-Yuan, Taiwan; Department of Biomedical Imaging and Radiological Sciences (C.H.T, C.-P.L.) and Laboratory of Brain Connectivity (C.-P.L.), Institute of Neuroscience, National Yang-Ming University, Taipei, Taiwan; and Department of Radiology (M.C), University of North Carolina School of Medicine, Chapel Hill, North Carolina.

This work was partly supported by grants from the National Science Council (NSC982314-B-182A-051-MY3 to C.H. Toh and NSC98-2923-B-010-001-MY3 to C.-P. Lin) and the National Health Research Institute (NHRI-EX99-9813EC to C.-P. Lin).

The authors also acknowledge support from the Animal Molecular Imaging Center, Chang Gung Memorial Hospital at Linkou, and the MRI Core Laboratory of National Yang-Ming University, which was funded from the Ministry of Education of Taiwan (Aim for the Top University Plan).

Please address correspondence to Ching-Po Lin, PhD, Department of Biomedical Imaging and Radiological Sciences, Institute of Neuroscience, National Yang-Ming University, 155 Li-Nong St, Sec 2, Peitou, Taipei, Taiwan, Republic of China; e-mail: cplin@ym.edu.tw

Indicates open access to non-subscribers at www.ajnr.org

Indicates article with supplemental on-line tables

http://dx.doi.org/10.3174/ajnr.A2581 that can measure directional variation of water diffusivity for a given voxel by using metrics that quantify diffusion anisotropy, tensor orientation, or tensor shapes. DWI, which may be considered a limited form of DTI, measures overall diffusivity in terms of directionally averaged ADC and, in past investigations, has allowed differentiation of abscesses from glioblastomas and metastases, given that ADC is typically low in abscess cavities and high in tumor cysts. However, high diffusivity similar to that found in necrotic tumors has been reported in $5 \%-21 \%$ of abscesses that were untreated ${ }^{1,2}$ or treated conservatively. ${ }^{3,4}$ On the other hand, metastatic brain tumors $^{5-7}$ and glioblastomas ${ }^{1,8,9}$ with low ADC mimicking abscesses have also been reported. In short, differentiation of brain abscesses from necrotic glioblastomas and cystic metastases with DWI can sometimes be difficult.

FA quantifying diffusion anisotropy and geometric tensor metrics summarizing distribution of tensor shapes-categorized as $C_{l}, C_{p}$, and $C_{s}$ - derived from DTI can reflect the directional organization of tissue microstructure, which may not be limited to the white matter tracts alone. In a lesion in which normal white matter is absent or destroyed, DTI can reflect the histologic components. It has been shown that DTI is able to differentiate lymphomas from glioblastomas ${ }^{10}$ and 
classic meningiomas from atypical meningiomas, ${ }^{11}$ presumably due to the differences in cellularity and histologic architecture.

At pathologic examination, the cystic part of glioblastomas and metastases contains necrotic tumor tissue, while the enhancing rim represents viable tumor cells. On DTI, the FA values measured in the necrotic and solid enhancing parts of glioblastomas and metastases were typically lower than those in normal white matter. ${ }^{12,13} \mathrm{~A}$ rim of brain tissue with high anisotropy in the peritumoral edema surrounding glioblastomas and metastases has been observed. ${ }^{14}$ For brain abscess, the cavity contains necrotic debris, neutrophils, and bacteria, whereas the enhancing rim is a fibrous capsule formed by collagen. ${ }^{15,16}$ Some studies ${ }^{17-20}$ have shown that FA in the cystic cavity and enhancing rim of an abscess can be as high as that found in normal white matter. In contrast, to our knowledge, high anisotropy has not been reported in the perifocal edema of brain abscess.

On the basis of the DTI findings from the published literature, we hypothesized that diffusion properties as detected by DTI can reflect the differences in histologic components of abscess, glioblastoma, and metastasis. We aimed to differentiate abscesses from glioblastomas and metastases with DTI and to compare the diagnostic performance of FA, geometric tensor metrics, and ADC.

\section{Materials and Methods}

\section{Patients}

Approval for this study was obtained from the Institutional Board of Research Associates, and signed informed consent was obtained from all patients. This study complied with the Health Insurance Portability and Accountability Act. No patients had begun corticosteroid or antibiotic treatment at the time of MR imaging. Preoperative MR imaging studies were performed in 15 patients ( 9 men, 6 women; mean age, 55.7 years; age range, $24-79$ years) with brain abscesses (mean size, $3.9 \pm 1.1 \mathrm{~cm}$ ), 15 patients ( 10 men, 5 women; mean age, 60.9 years; age range, $38-81$ years) with necrotic glioblastomas (mean size, $4.9 \pm 1.5 \mathrm{~cm}$ ), and 26 ( 14 men, 12 women; mean age, 58.5 years; age range, 21-79 years) patients with solitary cystic metastatic brain tumors (mean size, $3.1 \pm 1.0 \mathrm{~cm}$ ). All lesions appeared as a rimenhancing mass with perifocal edema on MR imaging. Lesions with large areas of susceptibility thought to be related to calcifications and/or hemorrhages were excluded. The diagnosis of brain abscess was confirmed on surgery in all patients. Histologic diagnosis was obtained in all patients with glioblastomas and metastases by surgical resection. In patients with brain metastases, the primary tumors included 13 lung carcinomas, 4 gastrointestinal carcinomas, 7 breast cancers, and 2 from unknown primary sites.

\section{MR Imaging}

All patients underwent MR imaging studies including axial T1weighted imaging, axial T2-weighted imaging, DTI, and postcontrast 3D MPRAGE with intravenous administration of $0.1 \mathrm{mmol}$ per kilogram of body weight of gadopentetate dimeglumine (Magnevist; Schering, Berlin, Germany). All MR imaging studies were performed on a single occasion by using a $3 \mathrm{~T}$ unit (Magnetom Tim Trio; Siemens, Erlangen, Germany). DTI was performed in the axial plane by using single-shot echo-planar imaging with the following parameters:
TR/TE, 5800/83 ms; diffusion-gradient encoding in 20 directions; $b=0,1000 \mathrm{~s} / \mathrm{mm}^{2}$; FOV, $256 \times 256 \mathrm{~mm}$; matrix size, $128 \times 128$; section thickness, $2 \mathrm{~mm}$; and number of signals acquired, 4 . A total of $50-60$ sections without intersection gap were used to cover the cerebral hemispheres, upper brain stem, and cerebellum. To minimize artifacts such as signal intensity drop-out and gross geometric distortions associated with the echo-planar imaging, we used parallel imaging technique (generalized autocalibrating partially parallel acquisition; reduction factor $=2$ ) during DTI acquisitions.

\section{Image Postprocessing}

Diffusion tensor data were transferred to an independent workstation and processed by using the software nordicICE (Nordic Image Control and Evaluation, Version 2.2; Nordic Imaging Lab, Bergen, Norway). The DWIs were coregistered to the non-DWIs $(b=0)$ to minimize the artifacts induced by eddy current and subject motion. The diffusion tensor was diagonalized to yield the major $\left(\lambda_{1}\right)$, intermediate $\left(\lambda_{2}\right)$, and minor $\left(\lambda_{3}\right)$ eigenvalues. FA, geometric tensor metrics $\left(C_{l}, C_{p}\right.$, and $\left.C_{s}\right)$, and ADC were calculated by using the following standard algorithms respectively:

$$
\begin{aligned}
& \mathrm{ADC}=\bar{\lambda}=\frac{\lambda_{1}+\lambda_{2}+\lambda_{3}}{3}, \\
& \mathrm{FA}=\sqrt{\frac{3}{2}} \cdot \sqrt{\frac{\left(\lambda_{1}-\bar{\lambda}\right)^{2}+\left(\lambda_{2}-\bar{\lambda}\right)^{2}+\left(\lambda_{3}-\bar{\lambda}\right)^{2}}{\lambda_{1}{ }^{2}+\lambda_{2}{ }^{2}+\lambda_{3}{ }^{2}}}, \\
& C_{l}=\frac{\lambda_{1}-\lambda_{2}}{\lambda_{1}+\lambda_{2}+\lambda_{3}}, \\
& C_{p}=\frac{2 \cdot\left(\lambda_{2}-\lambda_{3}\right)}{\lambda_{1}+\lambda_{2}+\lambda_{3}},
\end{aligned}
$$

and

$$
C_{s}=\frac{3 \lambda_{3}}{\lambda_{1}+\lambda_{2}+\lambda_{3}} .
$$

By using the coregistration module integrated in the software nordicICE, the tensor maps and T2-weighted images were coregistered to postcontrast MPRAGE on the basis of 3D nonrigid transformation and mutual information. The adequacy of registration was visually assessed by a neuroradiologist, and manual adjustments were performed if necessary. On the basis of the T2-weighted imaging and postcontrast MPRAGE, each lesion was manually segmented section by section into 4 nonoverlapping regions: cystic cavity, enhancing rim, immediate zone of edema (edema most adjacent to the enhancing rim), and distant zone of edema. A polygonal region of interest was first drawn to include the entire enhancing lesion. By manual pixel thresholding, we created a scatter region of interest to segment the entire enhancing rim. Then the cystic cavity and immediate and distant zones of edema were segmented sequentially. To avoid overlapping of the regions of interest, we nulled all pixels within each created region of interest before segmentation of the next one. For each lesion, a circular region of interest was placed in the contralateral deep white matter to measure the tensor metrics. The segmentation of region of interest and tensor metric measurements was performed by a neuroradiologist.

Two neuroradiologists who were blinded to the subjects evaluated postcontrast MPRAGE images and FA maps of all patients independently. Differences were resolved by consensus. In the perifocal edema, a hyperintense FA rim was evaluated as present if it was seen in more than two-thirds of sections covering the entire lesion or absent 

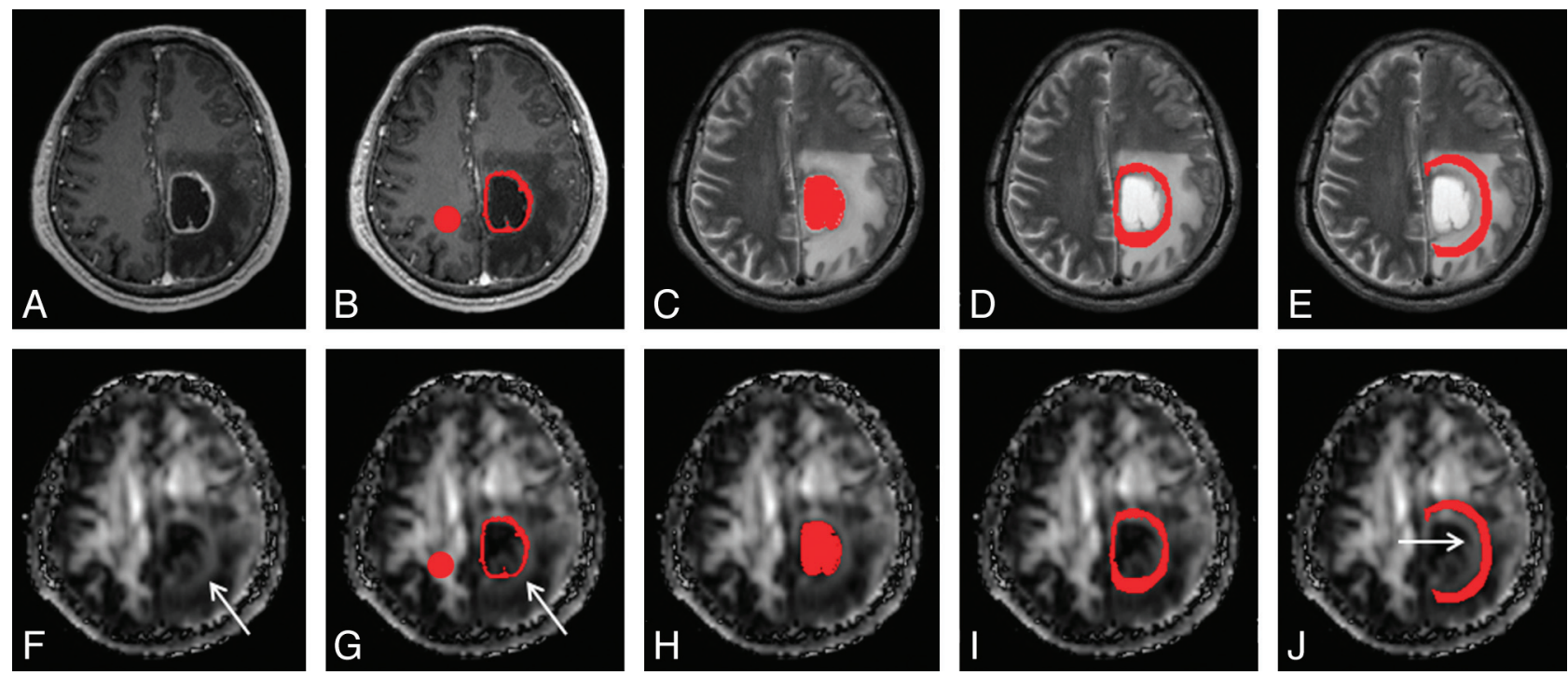

Fig 1. An example of how the regions of interest were segmented in a left frontal glioblastoma. Four regions of interest (ie, enhancing rim $[B$ and $G]$, cystic cavity [ $C$ and $H$, and immediate $[D$ and $I$ and distant zones of edema [ $E$ and $J]$ ) were segmented with contrast-enhanced MPRAGE and T2-weighted images (upper panel) and then were overlaid on FA maps (lower panel). Note that there is a rim of high FA in the immediate zone of edema (arrows in $F, G$, and $\mathcal{J}$. A circular region of interest is placed on the contralateral NAWM (B and G).

if found in less than one-third of sections. The immediate zone of edema would include only the hyperintense FA rim if it was present or was arbitrarily chosen as a 6-mm-wide band, which was the mean width of the hyperintense FA rim in this study. The distant zone of edema would have approximately the same width as the immediate zone of edema. An example of all regions of interest is shown in Fig 1.

\section{Statistical Analysis}

The incidence of a hyperintense FA rim in the perifocal edema of abscesses, glioblastomas, and metastases was examined by using a $\chi^{2}$ test. The mean FA, ADC, $C_{l}, C_{p}$, and $C_{s}$ values for each region of interest were compared by using analysis of variance among abscesses, glioblastomas, and metastases. Multiple pair-wise comparisons were performed with the Scheffe test. Cutoff values of FA, ADC, $C_{l}, C_{p}$, and $C_{s}$ for distinguishing abscesses from glioblastomas and metastases were determined by ROC curve analysis. The ROC curve was further used to calculate the AUC value, which is an index of overall discriminative ability of a given method. A commercially available statistical software package (Statistical Package for the Social Sciences, Version 17; SPSS, Chicago, Illinois) was used for analysis, and $P$ values $<.05$ were considered statistically significant.

\section{Results}

The diffusion tensor metric values derived from all regions of interest and the statistical results of between-group comparisons are listed in On-line Table 1.

\section{Cystic Cavity}

The FA, ADC, $C_{l}, C_{p}$, and $C_{s}$ values of the abscess cavity were significantly different from those measured in the cystic cavity of glioblastomas and metastases, respectively. The abscess cavity had significantly higher FA, lower ADC, higher linear tensor, higher planar tensor, and lower spheric tensor values compared with the cystic cavity of glioblastomas and metastases. None of the tensor metrics were different between glioblastomas and metastases.

\section{Enhancing Rim}

There were significantly higher FA, lower ADC, higher planar tensor, and lower spheric tensor values in the enhancing rim of abscesses than in glioblastomas. There was no significant difference in the linear tensor values between the 2 . All tensor metrics measured in the enhancing rim were significantly different between abscess and metastasis. Abscesses had significantly higher FA, lower ADC, higher linear tensor, higher planar tensor, and lower spheric tensor values compared with metastases.

Among the 4 regions of interest, the enhancing rim was the only region with tensor metrics that could differentiate glioblastoma from metastasis. Glioblastoma had significantly higher FA, higher linear tensor, higher planar tensor, and lower spheric tensor values compared with metastasis. The $\mathrm{ADC}$ of the enhancing rim was not different between glioblastoma and metastasis.

\section{Edema and Contralateral NAWM}

All tensor metrics in the immediate zone of edema were significantly different between abscess and glioblastoma. Brain abscess has lower FA, higher ADC, lower linear tensor, lower planar tensor, and higher spheric tensor values compared with glioblastoma in the immediate zone of edema. Compared with metastasis, abscess had significantly lower FA, higher ADC, and lower linear tensor values in the immediate zone of edema. No tensor metrics were different between glioblastoma and metastasis in the immediate zone of edema. None of the tensor metrics in the distant zone of edema and contralateral NAWM were different among abscess, glioblastoma, and metastasis.

\section{Visual Inspections of FA Maps}

A hyperintense FA rim in the immediate zone of edema was observed in 2 of 15 brain abscesses (13.3\%), 14 of 15 glioblastomas $(93.3 \%)$, and 22 of 26 metastases $(84.6 \%)$ 


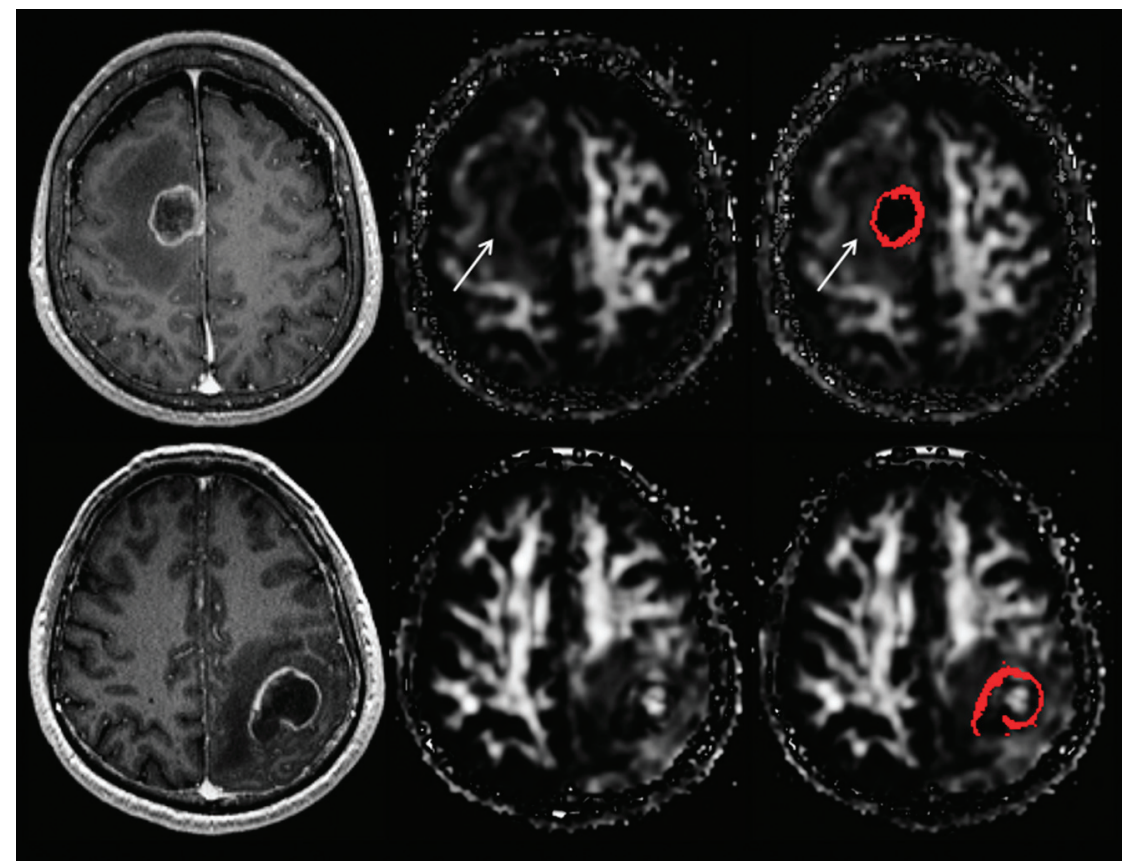

Fig 2. Visual assessment for the presence of a hyperintense FA rim in the immediate zone of edema. The upper panel shows the contrast-enhanced MPRAGE image, FA, and FA with scattered regions of interest representing the enhancing rim of a metastatic brain tumor (from left to right); the lower panel shows the corresponding images from a brain abscess. A hyperintense FA rim is present in the immediate zone of edema surrounding the metastatic tumor (arrow) but absent from that of the abscess.

(Figs 1 and 2). The incidence was significantly higher in glioblastomas $(P<.001$, odds ratio $=91)$ and metastases $(P<.001$, odds ratio $=35.7)$ compared with abscesses. The sensitivity, specificity, accuracy, positive predictive value, negative predictive value, and likelihood ratio of the hyperintense FA rim in differentiating abscess from glioblastoma and metastasis were $87.8 \%, 86.7 \%, 87.5 \%, 94.7 \%, 72.2 \%$, and $6.6 \%$, respectively. On the other hand, the incidence was not different between glioblastoma and metastasis $(P=$ $.393)$.

\section{Diagnostic Performance of the Tensor Metrics}

The diagnostic performance of tensor metrics for each region of interest in differentiating abscess from glioblastoma multiforme and metastasis is presented in On-line Table 2. The 3 tensor metrics having the highest sensitivity were FA, ADC, and $C_{l}$ measured in the cystic cavity. FA and $C_{s}$ of the cystic cavity as well as FA of the enhancing rim were the 3 with the highest specificity and accuracy. In terms of overall performance as determined by AUC, the 3 metrics with the highest performance were $\mathrm{FA}, C_{l}$, and $C_{s}$ of the cystic cavity.

\section{Discussion}

Our study shows that abscess was significantly different from glioblastoma and metastasis on quantitative and qualitative DTI analysis of their cystic cavities, enhancing rims, and perifocal edema, respectively. These differences indicate different patterns of water diffusion that allow discrimination of abscess from glioblastoma and metastasis.

In this study, the abscess cavity had higher FA compared with the cystic cavity of glioblastomas and metastases. The higher FA was postulated to reflect organized and oriented viable inflammatory cells ${ }^{21}$ in the abscess cavity due to up- regulation of various adhesion surface molecules. We found that the increase of both linear and planar tensors led to higher FA in the abscess cavity, with greater contribution from the former. Furthermore, the abscess cavity was the only region of interest that was found to have higher linear tensor than planar tensor values. It appeared that up-regulation of inflammation molecules in the abscess cavity predominantly increased the linear tensor values and, to a lesser extent, the planar tensor values. However, a previous study ${ }^{18}$ showed the reverse findings in that there were higher planar than linear tensor values in the abscess cavity. We believe the contradictory results are due to different region-of-interest selection methods between the 2 studies. Unlike the previous study ${ }^{18}$ excluding voxels with FAs $<0.2$, we included all voxels in the abscess cavity during quantitative analysis because the histologic components for voxels with $\mathrm{FA}<0.2$ were not clear; thus, this process made our study less susceptible to selection bias.

In this study, FA values were higher in the enhancing rim of the abscess than in that of glioblastoma and metastasis. The higher FA values in the abscess rim were predominantly contributed by increased planar tensor values, which may be secondary to the presence of concentric layers of collagen fibers that intermixed with the neutrophils and macrophages. ${ }^{15,19}$ To the best of our knowledge, there has been only 1 study comparing the enhancing rim of abscess and glioblastoma with DTI. By using random manual region-of-interest placement, the previous study reported no difference in FA values between abscess and glioblastoma. ${ }^{9}$ There are several differences between the present study and that one. First, this study had larger patients groups: 15 abscesses and 15 glioblastomas compared with 5 abscesses and 9 glioblastomas for the previous study. Second, there were several techniques used in our study that provided key improvements toward successful differentiation of abscess from glioblastoma. Manual placement 
of a region of interest in the thin enhancing rim, as performed in the previous study, would lead not only to inherent subjectivity but also partial volume effects by the inclusion of voxels from the adjacent cystic cavity and perifocal edema. To overcome these issues, we applied rigorous image registration and segmentation with scatter region of interest for all investigated regions, to enhance the accuracy of tensor metrics measurement.

In previous reports, a rim of high planar tensors in the immediate zone of edema was observed in 23 of 30 meningiomas (77\%), ${ }^{22} 25$ of 38 glioblastomas (66\%), and 15 of 25 metastases $(60 \%) .{ }^{14}$ By visual assessment of the FA map, which is more popular clinically, we found that the hyperintense rim was present in $93.3 \%$ of glioblastomas and $84.6 \%$ of metastases and its incidence was higher than that reported in the planar tensor map. We believe the higher incidence in the FA map was due to additional contributions from the linear tensor in addition to the planar tensor.

In contrast, the hyperintense FA rim was found only in $13.3 \%$ of abscesses in our study. The lower incidence of a hyperintense FA rim was concurrently supported by quantitative analysis showing that FA was significantly lower in the immediate zone of the edema of abscess than in glioblastoma multiforme and metastasis. To the best of our knowledge, we are the first to report the qualitative and quantitative differences in the immediate zone of edema on DTI, which allow differentiating abscess from glioblastoma and metastasis. We further demonstrated that visual assessment for the presence of a hyperintense FA rim in the immediate zone of edema provided higher sensitivity, specificity, and accuracy than quantitative measurement of the tensor metrics in differentiating abscess from glioblastoma and metastasis and, therefore, could be applied in routine clinical practice easily.

In previous reports, the increase of planar tensors in the immediate peritumoral region was considered nonspecific and simply due to the compression of surrounding tissues. ${ }^{14,22,23}$ In the present study, however, a hyperintense FA rim was hardly found in the edema of abscesses, even though many were large and associated with significant mass effect. It was only seen in 2 abscesses in the basal ganglia and was likely from partial volume of the internal capsule. In the immediate zone of edema of glioblastoma and metastasis, the linear and planar tensor values were higher compared with those of abscess, indicating more organized water diffusion. Therefore, we speculate that the presence of a hyperintense FA rim in the immediate zone of edema was not simply a compressive effect. Rather, it may suggest that microstructural changes in the edematous white matter immediately surrounding the abscess may be different from those surrounding glioblastoma and metastasis.

Gliosis is an astrocytic response to any central nervous system injury, which can occur in the perifocal edema of abscess, glioblastoma, and metastasis. ${ }^{15}$ The development of an encapsulated abscess and white matter edema usually takes about 2 weeks or less. Therefore, the edema and the gliotic response surrounding the abscess exist for a shorter duration, and the gliosis may be in an earlier stage than ones surrounding glioblastoma and metastasis. We speculate that glial fibers are laid down more irregularly in the early stage of gliosis found in the immediate zone of edema of the abscess. In the relatively long- standing edema and gliosis surrounding glioblastoma and metastasis, which take months to develop, glial fibers may assume a more regular arrangement, resulting in more organized water diffusion as detected with DTI. Further studies of the micro- or even ultrastructure of edema surrounding abscess, glioblastoma, and metastasis are needed to support our speculation.

Our results clearly showed that in differentiating abscess from glioblastoma and metastasis, tensor metrics measuring diffusion anisotropy (ie, FA) and tensor shapes (ie, $C_{l}$ and $C_{s}$ ) outperformed ADC in measuring mean diffusivity. The differences in diagnostic performance of the tensor metrics reflect the ability of a particular tensor metric in delineating the histopathologic differences between abscess, glioblastoma, and metastasis.

There are many factors such as cellularity, viscosity, permeability, and histologic architecture that affect water diffusivity. The alteration of mean and directional variation of water diffusivity as measured with DTI is the summation effect of all these microstructural barriers. Because in vivo quantification of the individual effect of each factor is currently impossible, we could not definitely state that the changes of tensor metrics were directly related to the differences in histologic features such as the number of inflammatory molecules, concentric arrangement of collagen fibers, and the microstructure of gliotic responses. This is a limitation of our study but is present in most of the research involving in vivo tensor metrics measurement. Despite the inherent limitation of DTI, abscess is unequivocally different from glioblastoma and metastasis on quantitative and qualitative DTI analysis in our study.

\section{Conclusions}

Abscess was significantly different from glioblastoma and metastasis in the analysis of the cystic cavity, enhancing rim, and immediate zone of edema with DTI. For a rim-enhancing mass, the presence of a hyperintense FA rim in the immediate zone of edema favored the diagnosis of glioblastoma or metastasis. DTI is able to differentiate abscess from glioblastoma and metastasis with qualitative assessment and quantitative measurement. Tensor metrics measuring diffusion anisotropy or tensor shapes outperformed ADC in differentiating abscess from glioblastoma and metastasis.

\section{References}

1. Reddy JS, Mishra AM, Behari S, et al. The role of diffusion-weighted imaging in the differential diagnosis of intracranial cystic mass lesions: a report of 147 lesions. Surg Neurol 2006;66:246-50

2. Lee EJ, Ahn KJ, Ha YS, et al. Unusual findings in cerebral abscess: report of two cases. Br J Radiol 2006;79:e156-61

3. Cartes-Zumelzu FW, Stavrou I, Castillo M, et al. Diffusion-weighted imaging in the assessment of brain abscesses therapy. AJNR Am J Neuroradiol 2004;25: 1310-17

4. Mishra AM, Gupta RK, Jaggi RS, et al. Role of diffusion-weighted imaging and in vivo proton magnetic resonance spectroscopy in the differential diagnosis of ring-enhancing intracranial cystic mass lesions. J Comput Assist Tomogr 2004;28:540-47

5. Holtas S, Geijer B, Stromblad LG, et al. A ring-enhancing metastasis with central high signal on diffusion-weighted imaging and low apparent diffusion coefficients. Neuroradiology 2000;42:824-27

6. Hartmann M, Jansen $\mathrm{O}$, Heiland $\mathrm{S}$, et al. Restricted diffusion within ring enhancement is not pathognomonic for brain abscess. AJNR Am J Neuroradiol 2001;22:1738-42

7. Tung GA, Evangelista P, Rogg JM, et al. Diffusion-weighted MR imaging of 
rim-enhancing brain masses: is markedly decreased water diffusion specific for brain abscess? AJR Am J Roentgenol 2001;177:709-12

8. Hakyemez B, Erdogan C, Yildirim N, et al. Glioblastoma multiforme with atypical diffusion-weighted MR findings. Br J Radiol 2005;78:989-92

9. Reiche W, Schuchardt V, Hagen T, et al. Differential diagnosis of intracranial ring enhancing cystic mass lesions: role of diffusion-weighted imaging (DWI) and diffusion-tensor imaging (DTI). Clin Neurol Neurosurg 2010;112:218-25

10. Toh $\mathrm{CH}$, Castillo M, Wong AM, et al. Primary cerebral lymphoma and glioblastoma multiforme: differences in diffusion characteristics evaluated with diffusion tensor imaging. AJNR Am J Neuroradiol 2008;29:471-75

11. Toh $\mathrm{CH}$, Castillo M, Wong AM, et al. Differentiation between classic and atypical meningiomas with use of diffusion tensor imaging. AJNR Am J Neuroradiol 2008;29:1630-35

12. Sinha S, Bastin ME, Whittle IR, et al. Diffusion tensor MR imaging of highgrade cerebral gliomas. AJNR Am J Neuroradiol 2002;23:520-27

13. Wang W, Steward CE, Desmond PM. Diffusion tensor imaging in glioblastoma multiforme and brain metastases: the role of $\mathrm{p}, \mathrm{q}, \mathrm{L}$, and fractional anisotropy. AJNR Am J Neuroradiol 2009;30:203-08

14. Wang S, Kim S, Chawla S, et al. Differentiation between glioblastomas and solitary brain metastases using diffusion tensor imaging. Neuroimage 2009;44: 653-60

15. Ellison D, Love S, Chimelli L, et al. Neuropathology: A Reference Text of CNS Pathology. 2nd ed. Edinburgh, United Kingdom: Mosby; 2004:xii, 755
16. Britt RH, Enzmann DR, Yeager AS. Neuropathological and computerized tomographic findings in experimental brain abscess. J Neurosurg 1981;55:590603

17. Gupta RK, Hasan KM, Mishra AM, et al. High fractional anisotropy in brain abscesses versus other cystic intracranial lesions. AJNR Am J Neuroradiol 2005 26:1107-14

18. Kumar M, Gupta RK, Nath K, et al. Can we differentiate true white matter fibers from pseudofibers inside a brain abscess cavity using geometrical diffusion tensor imaging metrics? NMR Biomed 2008;21:581-88

19. Gupta RK, Srivastava S, Saksena S, et al. Correlation of DTI metrics in the wall and cavity of brain abscess with histology and immunohistochemistry. NMR Biomed 2010;23:262-69

20. Mishra AM, Gupta RK, Saksena S, et al. Biological correlates of diffusivity in brain abscess. Magn Reson Med 2005;54:878-85

21. Gupta RK, Nath K, Prasad A, et al. In vivo demonstration of neuroinflammatory molecule expression in brain abscess with diffusion tensor imaging. AJNR Am J Neuroradiol 2008;29:326-32

22. Tropine A, Dellani PD, Glaser M, et al. Differentiation of fibroblastic meningiomas from other benign subtypes using diffusion tensor imaging. J Magn Reson Imaging 2007;25:703-08

23. Zhang S, Bastin ME, Laidlaw DH, et al. Visualization and analysis of white matter structural asymmetry in diffusion tensor MRI data. Magn Reson Med 2004;51:140-47 